\title{
A Cellular-Space-Division-Based Method of Moments Algorithm for the Pattern Analysis of Printed-Circuit Radiators
}

\author{
Martin Gimersky, Member, IEEE, and Jens Bornemann, Senior Member, IEEE
}

\begin{abstract}
A cellular-space-division-based method of moments (MoM) algorithm for the analysis of geometries involving imperfectly conducting planar radiators as well as lossy and finiteextent dielectric substrates is presented. Since the technique-via the volume equivalence theorem-replaces the structure under analysis with an equivalent structure composed of thin-wall cells, modeling of the surrounding environment is not required, hence, completely avoiding the need for absorbing boundary conditions. Real (as opposed to perfect) material parameters are incorporated via properly defined surface impedances. Several examples of radiation patterns (including radiation underneath the ground plane of a finite-extent substrate) of planar geometries are presented. The calculated patterns are compared with measured results and are found to be in good agreement.
\end{abstract}

Index Terms-Method of moments, microstrip antennas.

\section{INTRODUCTION}

$\mathbf{M}$ ODERN planar antenna designs rely heavily on accurate and reliable analysis software. Whereas increasing complexity of printed-circuit radiators demands a high flexibility from the underlying modeling tool, individual numerical techniques are often too restricted in their applications or too CPU time and memory intensive to allow an individual analysis or design to be completed in a timely fashion.

Desirable features of suitable numerical models include:

1) the capability of analyzing multilayered circuitry consisting of feed lines, apertures, radiator, and dielectric overlays (e.g., [1]);

2) the incorporation of dielectric and conductor losses (e.g., [2]);

3) the possibility of including finite-size and piecewise homogeneous dielectrics (e.g., [3]);

4) the limitation of the extent of a ground plane (e.g., [4]);

Manuscript received July 2, 1996; revised February 4, 1998.

M. Gimersky was with the Laboratory for Lightwave Electronics, Microwaves and Communications (LLiMiC), Department of Electrical and Computer Engineering, University of Victoria, Victoria, BC V8W 3P6, Canada. He is currently with Spar Aerospace Ltd., Ste. Anne-de-Bellevue, Quebec, H9X 3R2, Canada.

J. Bornemann is with the Laboratory for Lightwave Electronics, Microwaves and Communications (LLiMiC), Department of Electrical and Computer Engineering, University of Victoria, Victoria, BC V8W 3P6, Canada.

Publisher Item Identifier S 0018-926X(98)03421-8.

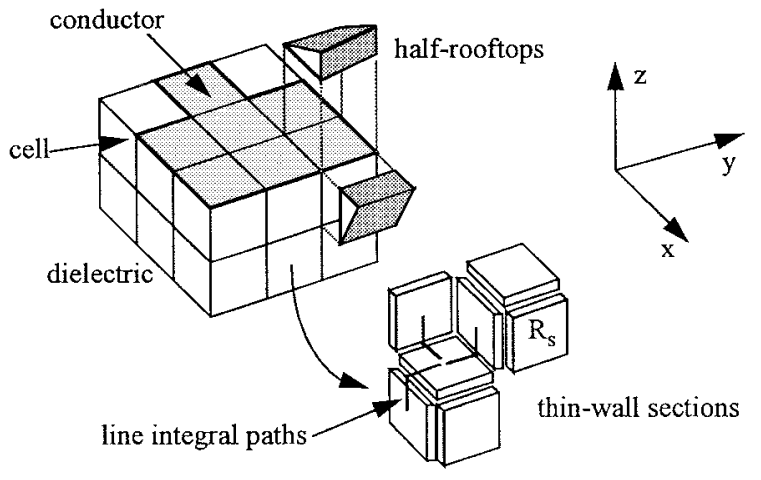

Fig. 1. Subdivision of a planar microwave structure into thin-wall sections.

5) the concentration of the numerical attention exclusively on the circuit (e.g., [5]) without the need for modeling the (free-space) environment and without any absorbing boundary conditions.

Therefore, this paper focuses on a cellular-space divisionbased method of moments (MoM) algorithm, which includes all the five features listed above. The method is primarily based on earlier work by Rubin and Daijavad [5]. However, we have made the method systematic by replacing the numerical integration procedure (which is the heart of the technique) with an exact, robust, and efficient algorithm [6], [7]. Moreover, we have implemented suggestions in [5] to incorporate conductor and substrate losses, which, nowadays, are considered a prerequisite for any meaningful printed-circuit analysis.

The fundamental steps are outlined in Section II below and include some modifications with respect to [5]. Section III details the numerical refinements for the implementation of the code. The results in Section IV focus on radiation patterns of planar antenna configurations for four types of feeding mechanisms. Results in terms of input impedance and input reflection coefficient for planar geometries are presented in [7] and [8] and need not be repeated here.

\section{THEORY}

Fig. 1 illustrates the subdivision of a planar microwave circuit into a cellular structure. Each of the $N_{x} \cdot N_{y} \cdot N_{z}$ thin-wall sections is associated with a series of full rooftop current functions, a full rooftop being composed of a rising half rooftop and a falling half rooftop [Fig. 2(a), (b)] [5]. For a 


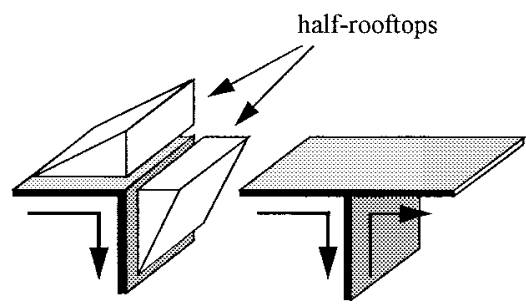

(a)

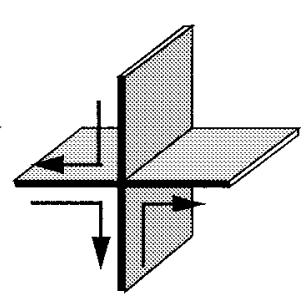

(c)
Fig. 2. Representation of junction currents. (a) At an external edge. (b) At a three junction. (c) At a four junction.

single cell where $N_{x}=N_{y}=N_{z}=1$, the number of currents required is 12 , but in practical applications the attachments to neighbor cells reduce the average number of currents (full rooftops) per cell $P_{\mathrm{av}}$ to

$$
\begin{aligned}
P_{\mathrm{av}}=9 & +2\left(\frac{1}{N_{x}}+\frac{1}{N_{y}}+\frac{1}{N_{z}}\right) \\
& -\left(\frac{1}{N_{x} N_{y}}+\frac{1}{N_{x} N_{z}}+\frac{1}{N_{y} N_{z}}\right)
\end{aligned}
$$

e.g., the fourth current in Fig. 2(c) is linearly dependent and, therefore, not shown. Although the number in (1) is larger than that of, e.g., a finite-difference analysis, it is noteworthy that the computational space is smaller, as we need not model the surrounding environment.

Material properties are incorporated through the cell's total impedances, e.g., $R_{x}$ in the $x$ direction. For the thin-wall structure, however, the surface impedance must be such that when multiplied by length $\tau_{x}$ and divided by perimiter $2\left(\tau_{y}+\right.$ $\tau_{z}$ ), the result is again $R_{x}$. Thus, the surface impedance along $x-R_{S x}$ (Fig. 3) -is given by

$$
R_{S x}=R_{x} \frac{2\left(\tau_{y}+\tau_{z}\right)}{\tau_{x}}=\frac{2\left(\frac{1}{\tau_{y}}+\frac{1}{\tau_{z}}\right)}{j \omega \epsilon_{0}\left(\epsilon_{r}-1\right)} \quad[\Omega]
$$

where expressions

$$
\begin{aligned}
\epsilon & =\epsilon^{\prime}-j \epsilon^{\prime \prime}=\epsilon_{0} \epsilon_{r}-j \frac{\sigma_{d}}{\omega} \quad[\mathrm{F} / \mathrm{m}] \\
R_{S x, y, z} & =\frac{1+j}{\delta \sigma_{m}} \quad[\Omega]
\end{aligned}
$$

are used for imperfect dielectrics and conductors, respectively. $\sigma_{d}$ is the conductivity of the dielectric, $\sigma_{m}$ the conductivity of the metallization and $\delta$ is the penetration depth. Note that the permittivity in (2), utilizing the volume equivalence theorem [9], is defined with respect to the surrounding free-space environment.

The electric field boundary condition applied over each dielectric cell wall and conductor surface is

$$
\boldsymbol{E}_{t}^{\text {scat }}-\boldsymbol{J}_{S} R_{S}=-\boldsymbol{E}_{t}^{\mathrm{inc}} \quad[\mathrm{V} / \mathrm{m}]
$$

where the incident field $E^{\text {inc }}$ is related to the applied voltage $V_{\beta}$ by [5]

$$
V_{\beta}=-\int_{u_{1 \beta}}^{u_{2 \beta}} \boldsymbol{E}^{\mathrm{inc}}(x, y, z) \cdot e_{u \beta} d u \quad[\mathrm{~V}]
$$

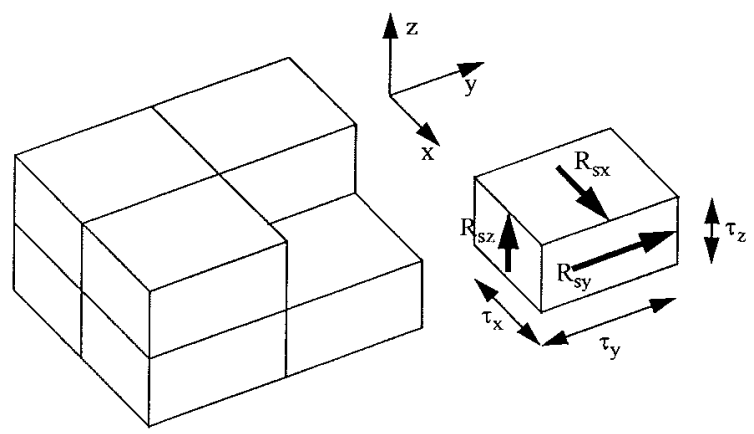

Fig. 3. Surface impedances on individual cells.

and the volume current density $\boldsymbol{J}$ may be compactly expressed as

$$
J=\sum_{\alpha=1}^{P} R_{\alpha}(x, y, z) I_{\alpha} \boldsymbol{e}_{u \alpha} \quad\left[\mathrm{A} / \mathrm{m}^{2}\right] .
$$

In (6), $I_{\alpha}[\mathrm{A} / \mathrm{m}]$ is the current coefficient, $P$ is the total number of rooftop functions, and

$$
R_{\alpha}(x, y, z)=q_{\tau_{u \alpha}}\left(u-u_{\alpha}\right) p_{\tau_{v \alpha}}\left(v-v_{\alpha}\right) \delta\left(w-w_{\alpha}\right) \quad[\mathrm{l} / \mathrm{m}]
$$

is the rooftop function centered at $x=x_{\alpha}, y=y_{\alpha}, z=z_{\alpha}$, where $q_{\tau}(u)$ is the triangle function defined as

$$
q_{\tau}(u)= \begin{cases}1-\left|\frac{u}{\tau}\right|, & -\tau \leq u \leq \tau \\ 0, & \text { elsewhere }\end{cases}
$$

$p_{\tau}(v)$ is the pulse function defined as

$$
p_{\tau}(v)= \begin{cases}1, & -\tau / 2 \leq v \leq \tau / 2 \\ 0, & \text { elsewhere }\end{cases}
$$

and $\delta(w)[1 / \mathrm{m}]$ is the Dirac delta function. Moreover, variables $u, v, w, \tau_{u \alpha}, \tau_{v \alpha}$, and $\tau_{w \alpha}$ have been defined in terms of $x, y$, and $z$ (c.f., Fig. 3) as [5]

$$
\begin{aligned}
u & =\boldsymbol{e}_{u \alpha} \cdot\left(x \boldsymbol{e}_{x}+y \boldsymbol{e}_{y}+z \boldsymbol{e}_{z}\right) \\
v & =\boldsymbol{e}_{v \alpha} \cdot\left(x \boldsymbol{e}_{x}+y \boldsymbol{e}_{y}+z \boldsymbol{e}_{z}\right) \\
w & =\boldsymbol{e}_{w \alpha} \cdot\left(x \boldsymbol{e}_{x}+y \boldsymbol{e}_{y}+z \boldsymbol{e}_{z}\right) \\
\tau_{u \alpha} & =\boldsymbol{e}_{u \alpha} \cdot\left(\tau_{x} \boldsymbol{e}_{x}+\tau_{y} \boldsymbol{e}_{y}+\tau_{z} \boldsymbol{e}_{z}\right) \\
\tau_{v \alpha} & =\boldsymbol{e}_{v \alpha} \cdot\left(\tau_{x} \boldsymbol{e}_{x}+\tau_{y} \boldsymbol{e}_{y}+\tau_{z} \boldsymbol{e}_{z}\right) \\
\tau_{w \alpha} & =\boldsymbol{e}_{w \alpha} \cdot\left(\tau_{x} \boldsymbol{e}_{x}+\tau_{y} \boldsymbol{e}_{y}+\tau_{z} \boldsymbol{e}_{z}\right)
\end{aligned}
$$

where $\boldsymbol{e}_{x}, \boldsymbol{e}_{y}$, and $\boldsymbol{e}_{z}$ are the unit vectors along the $x, y$, and $z$ direction, respectively; $\boldsymbol{e}_{u \alpha}$ is along the direction of current flow, $e_{w \alpha}$ is normal to the plane containing the rooftop, and $\boldsymbol{e}_{v \alpha}$ is orthogonal to both $\boldsymbol{e}_{u \alpha}$ and $\boldsymbol{e}_{w \alpha}$.

Associated with each rooftop $\alpha$ is a line interval or integration path (Fig. 1) over which the electric field is tested. This interval is defined by its end points located at $\left(u_{1 \alpha}, v_{\alpha}, w_{\alpha}\right)$ and $\left(u_{2 \alpha}, v_{\alpha}, w_{\alpha}\right)$, where

$$
\begin{aligned}
& u_{1 \alpha}=u_{\alpha}-\tau_{u \alpha} / 2 \\
& u_{2 \alpha}=u_{\alpha}+\tau_{u \alpha} / 2
\end{aligned}
$$


e.g., for a rooftop that lies in the $y-z$ plane and has current directed along the $y$ direction $R_{\alpha}(x, y, z)=\delta\left(x-x_{\alpha}\right) q_{\tau y}(y-$ $\left.y_{\alpha}\right) p_{\tau z}\left(z-z_{\alpha}\right)$ and $u_{1 \alpha}=y_{\alpha}-\tau_{y} / 2$ and $u_{2 \alpha}=y_{\alpha}+\tau_{y} / 2$.

With this in mind, we introduce the following integrals:

$$
\begin{aligned}
F_{A}= & \iint q_{\tau_{u \alpha}}\left(u^{\prime}-u_{\alpha}\right) p_{\tau_{v \alpha}}\left(v^{\prime}-v_{\alpha}\right) \\
& \cdot \frac{e^{-j k r\left(u-u^{\prime}, v-v^{\prime}, w-w_{\alpha}\right)}}{r\left(u-u^{\prime}, v-v^{\prime}, w-w_{\alpha}\right)} d u^{\prime} d v^{\prime} \quad[\mathrm{m}] \\
F_{S}^{f / r}= & \frac{1}{\tau_{u \alpha}} \iint p_{\tau_{u \alpha}}\left(u^{\prime}-u_{\alpha} \mp \frac{\tau_{u \alpha}}{2}\right) p_{\tau_{v \alpha}}\left(v^{\prime}-v_{\alpha}\right) \\
& \cdot \frac{e^{-j k r\left(u-u^{\prime}, v-v^{\prime}, w-w_{\alpha}\right)}}{r\left(u-u^{\prime}, v-v^{\prime}, w-w_{\alpha}\right)} d u^{\prime} d v^{\prime}
\end{aligned}
$$

where $f / r$ denotes the falling/rising half rooftop.

Since the scattered field can be written as $E^{\text {scat }}=-j \omega A-$ $\nabla \phi$ and since the total vector and scalar potentials are given by

$$
\begin{aligned}
& A=\frac{\mu_{0}}{4 \pi} \sum_{\alpha=1}^{P} I_{\alpha} \boldsymbol{e}_{u \alpha} F_{A} \\
& \Phi=\frac{1}{j 4 \pi \omega \epsilon_{0}} \sum_{\alpha=1}^{P} I_{\alpha}\left(F_{S}^{f}-F_{S}^{\boldsymbol{r}}\right)
\end{aligned}
$$

the individual entries $Z_{\beta \alpha}$ of the impedance matrix used in the MoM formulation are obtained as

$$
\begin{aligned}
Z_{\beta \alpha}=- & \int_{u_{1 \beta}}^{u_{2 \beta}}\left[\frac{1}{4 \pi}\left(j \omega \mu_{0} F_{A} \boldsymbol{e}_{u \alpha}+\frac{1}{j \omega \epsilon_{0}} \nabla\left(F_{S}^{f}-F_{S}^{r}\right)\right)\right. \\
& \left.+\frac{\tau_{v \alpha}}{2\left(\tau_{v \alpha}+\tau_{w \alpha}\right)} R_{S \alpha} R_{\alpha}^{\prime}(x, y, z) \boldsymbol{e}_{u \alpha}\right] \\
\cdot \boldsymbol{e}_{u \beta} d u & {[\Omega \mathrm{m}] }
\end{aligned}
$$

where

$$
R_{\alpha}^{\prime}(x, y, z)= \begin{cases}q_{\tau_{u \alpha}}\left(u-u_{\alpha}\right) p_{\tau_{v \alpha}}\left(v-v_{\alpha}\right), & w=w_{\alpha} \\ 0, & w \neq w_{\alpha}\end{cases}
$$

For a given voltage excitation vector, the coefficients $I_{\alpha}$ $[\mathrm{A} / \mathrm{m}]$ of the equivalent surface currents, as dictated by the volume equivalence theorem, are obtained by inverting the impedance matrix. Since a numerically stable integration procedure is used [7], the entries of the impedance matrix are well defined, and so are the individual currents. Circuit responses and radiation characteristics are then calculated from the currents. For all structures analyzed so far, convergence is reached with 12-20 cells per guided wavelength $\left(\lambda_{g}\right)$ in case of scattering parameter and input impedance analysis, while $8-12$ cells $/ \lambda_{g}$ suffice for radiation characteristics.

\section{NUMERICAL REFINEMENTS}

For all self impedances and some mutual impedances, the integrals of (12) and (13) contain singularities. Although integrations over rectangular regions are required, in both cases we perform a transformation to the polar coordinate system. This eliminates the singularities in the generalized two-dimensional exponential integrals and an exact and stable solution free of any "numerical tuning," is obtained. For details of this procedure, the reader is referred to [6] and [7].

For the pairs of $I_{\alpha}$ (source) and $V_{\beta}$ (target) that are distant from each other, [5] employs the point-source approximation in the evaluation of (12) and (13) of the corresponding $Z_{\beta \alpha}$ 's. Evaluating the $Z_{\beta \alpha}$ 's for short source-target distances exactly, we have assessed that the point-source approximation unnecessarily compromises accuracy of the calculations for a given discretization and, therefore, avoided the approximation completely. Instead, we have employed the algorithm desribed bellow.

The algorithm is based on the following rationale. Clearly, numerical evaluation of $F_{A}$ and $F_{S}$, which are defined in (12) and (13), is the most important factor dictating the amount of CPU time necessary for setting up the impedance matrix. Any reduction in processor time needed for the evaluation of $F_{A}$ and $F_{S}$ will lower overall requirements for computer resources. Having decided not to employ the point-source approximation (which would be the least time demanding - at the price of reduced accuracy), we define an auxiliary variable $T$ as

$$
T=\frac{e^{-j k \sqrt{\left[\left(x_{1}+x_{2}\right) / 2\right]^{2}+\left[\left(y_{1}+y_{2}\right) / 2\right]^{2}+a^{2}}}}{\sqrt{\left[\left(x_{1}+x_{2}\right) / 2\right]^{2}+\left[\left(y_{1}+y_{2}\right) / 2\right]^{2}+a^{2}}}
$$

where $x_{1}, x_{2}, y_{1}$, and $y_{2}$ are the lower and upper integration limits in (12) and (13). Parameter $a$ is the difference between the $z$ coordinates of the two planar surfaces of interest and, therefore, a constant with respect to the integration. With this notation, $F_{S}$ can be expressed as

$$
\begin{gathered}
F_{S}=\frac{1}{x_{2}-x_{1}}\left[T \cdot\left(x_{2}-x_{1}\right)\left(y_{2}-y_{1}\right)+\int_{x_{1}<0}^{x_{2}>0} \int_{y_{1}<0}^{y_{2}>0}\right. \\
\left.\cdot\left(\frac{e^{-j k \sqrt{x^{\prime 2}+y^{\prime 2}+a^{2}}}}{\sqrt{x^{\prime 2}+y^{\prime 2}+a^{2}}}-T\right) d y^{\prime} d x^{\prime}\right] .
\end{gathered}
$$

Similarly, $F_{A}$-for a falling half rooftop —can be written as

$$
\begin{aligned}
F_{A}= & 0.5 T \cdot x_{2} \cdot 2 y_{2}+\int_{0}^{x_{2}} \int_{-y_{2}}^{y_{2}} \\
& \cdot\left[\left(1-\frac{x^{\prime}}{x_{2}}\right) \frac{e^{-j k \sqrt{x^{\prime 2}+y^{\prime 2}+a^{2}}}}{\sqrt{x^{\prime 2}+y^{\prime 2}+a^{2}}}=0.5 T\right] d y^{\prime} d x^{\prime} .
\end{aligned}
$$

The calculation of $F_{A}$ for a rising half rooftop is analogous.

While [in comparison with (12) and (13)] there are extra additive and subtractive operations in (19) and (20), 


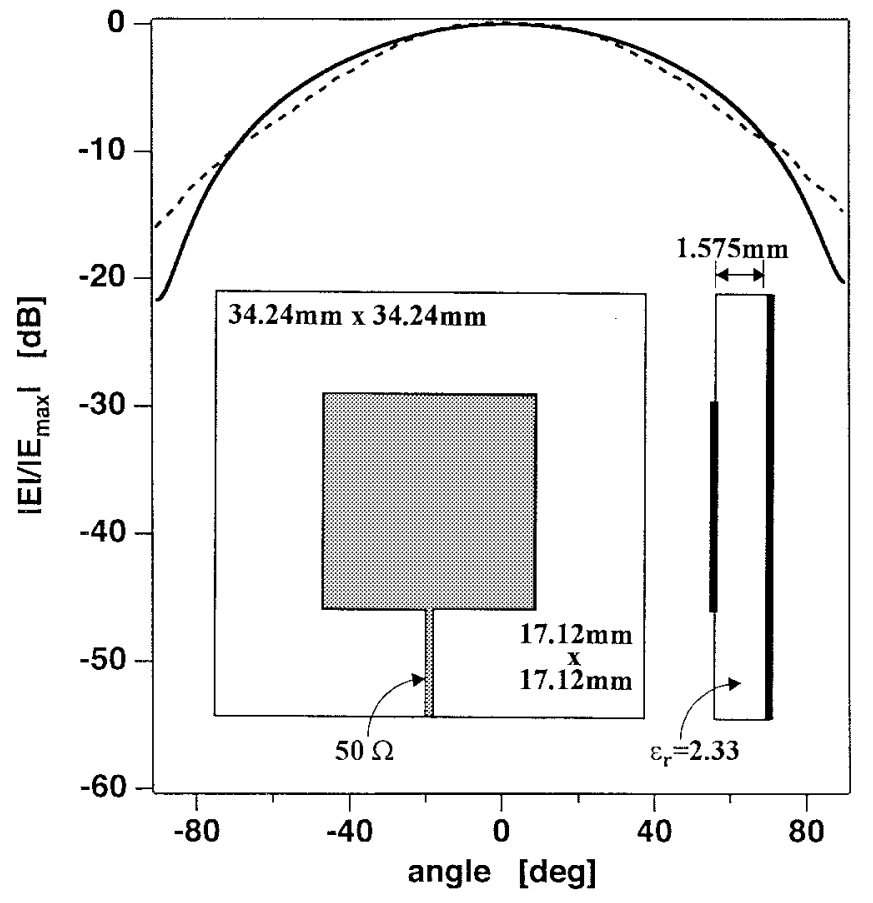

Fig. 4. $E$-plane radiation patterns of a symmetrically edge-fed square patch manufactured on copper-clad RT/Duroid at $5.3 \mathrm{GHz}$. Calculated (solid line); measured [11] (dashed line).

the integrands of (19) and (20) are significantly smoother functions than those of (12) and (13) for large values of $\sqrt{x^{\prime 2}+y^{\prime 2}+a^{2}}$. As a result, fewer integration points are needed.

Compared with the point-source approximation, the above procedure costs us some computational time but considerably improves the accuracy of the resulting current coefficients $I_{\alpha}$ and, therefore, the reliability of the computation.

\section{RESULTS}

The calculations presented in this section respect finite conductivities of metals and nonzero conductivities of dielectrics. To prevent computer memory and time requirements from becoming exuberant, however, metallic surfaces were modeled as having zero thicknesses.

The first example is a square patch symmetrically edge fed by a $50-\Omega$ microstrip transmission line, as shown in the inset of Fig. 4. The structure forms a single element of an array for synthetic aperture radar applications [10]. Although other circuitry (such as feed lines and matching transformers) is integrated on the substrate, it has not been modeled in order to limit the computational space. A minute asymmetry can be observed in both the calculated and measured [11] $E$ plane patterns. Inspection of the calculated current distribution confirms that the asymmetry is a result of the fact that the patch is slightly longer than one half of the guided wavelength at the given frequency. With the exception of angular values below $-80^{\circ}$ and above $80^{\circ}$, the calculated and measured patterns are in good agreement.

As a representative of a planar antenna fed by a coaxial probe, we present the arrangement depicted in the inset of

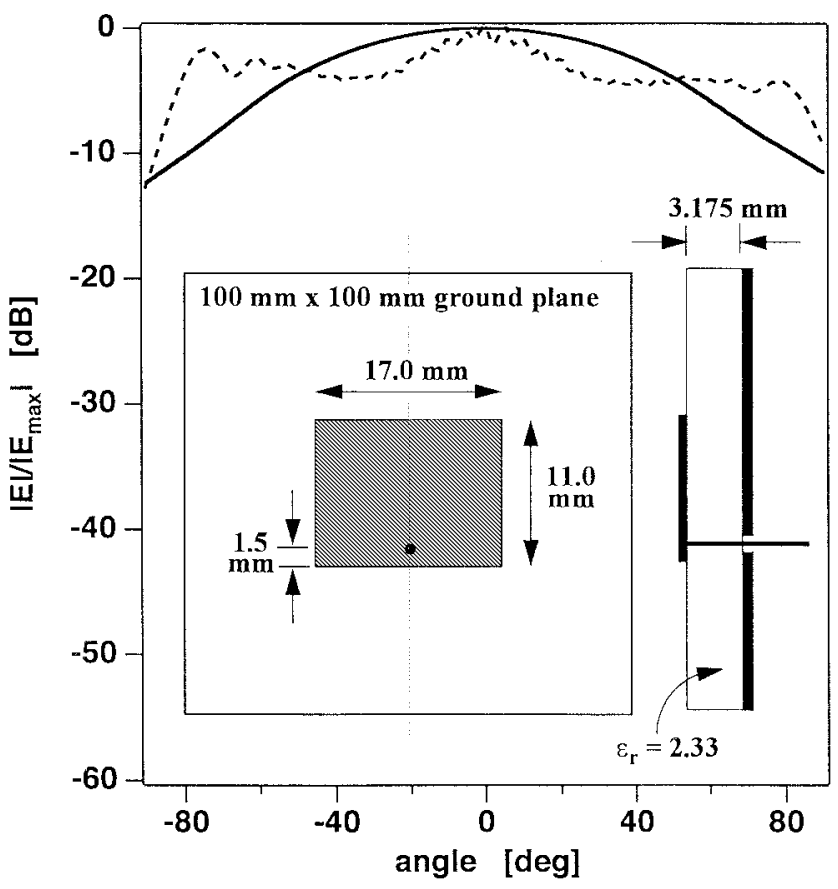

(a)

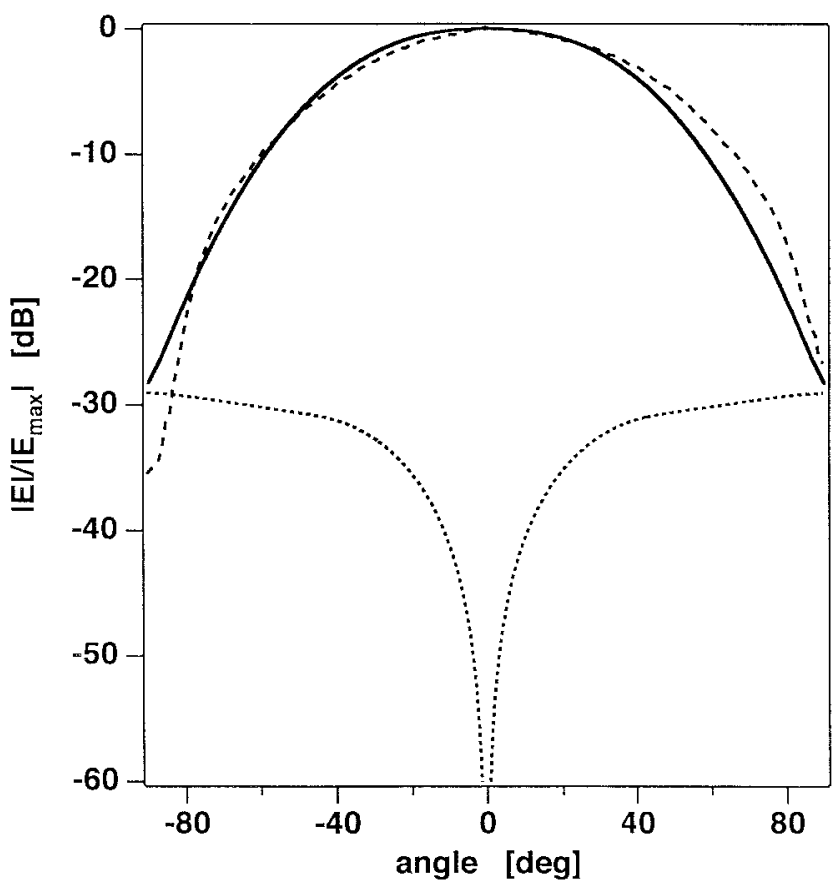

(b)

Fig. 5. Radiation patterns of the coaxially fed rectangular patch antenna [12] at $6.8 \mathrm{GHz}$. (a) $E$-plane-solid line: calculated copolarized; dashed line: measured copolarized [12]; calculated cross polarized below -100 dB. (b) $H$-plane- solid line: calculated copolarized; dashed line: measured copolarized [12]; dotted line: calculated cross polarized.

Fig. 5(a). Its measured [12] and calculated $E$ - and $H$-plane radiation patterns at $6.8 \mathrm{GHz}$ are shown in Fig. 5(a) and (b), respectively. Agreement between calculated and measured patterns is fairly good, especially in the $H$-plane. It is not clear whether the deviations in the $E$-plane are caused by measurement inaccuracies, the inability of our numerical 


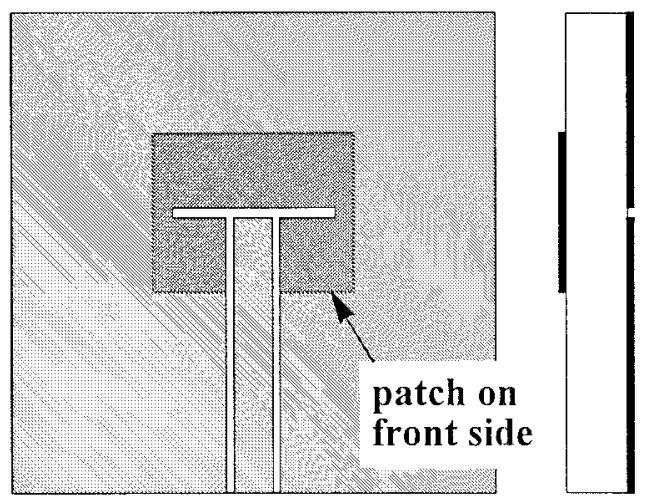

(a)

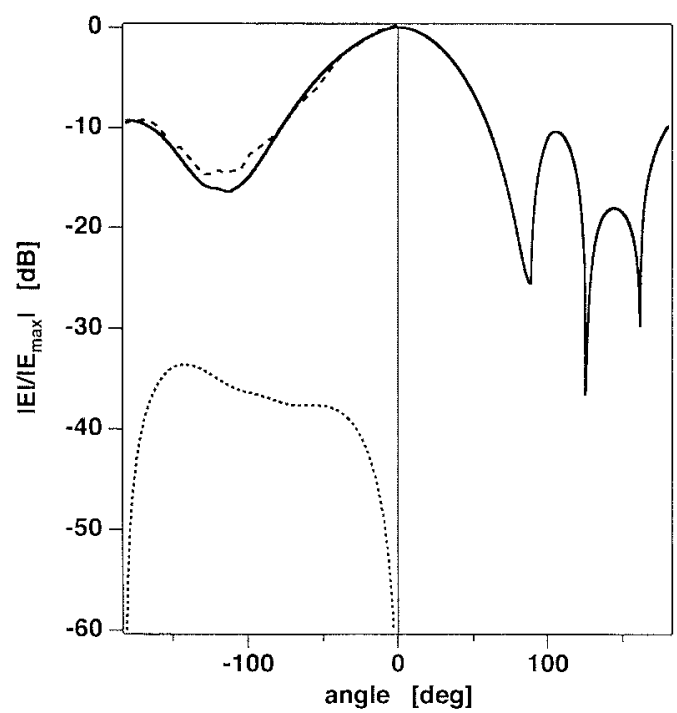

(b)

Fig. 6. Radiation patterns of the microstrip patch antenna with coplanar feeding at $4.3 \mathrm{GHz}$. Dimensions according to [13]. Substrate area modeled as nine times patch area. (a) Back-side view of substrate. (b) $H$-plane (left)—solid line: calculated copolarized; dashed line: measured copolarized [13]; dotted line: calculated cross polarized. $E$-plane (right)—solid line: calculated copolarized; calculated cross polarized below $-100 \mathrm{~dB}$.

model to represent circular (rather than square) via holes or a combination of both. (It might also be possible that the measurements in [12] have been conducted at the presence of a larger metallic plate attached to the gound plane.) Because the patch is fed symmetrically, the cross-polarized component of its $E$-plane radiation pattern should theoretically be zero. In this and the remaining examples, the calculated value is always below $-100 \mathrm{~dB}$. For the same reason as in the $E$-plane, the $H$-plane cross-polarized component should theoretically be zero at patch's zenith, i.e., at the angular value of zero degrees. In comparison, the calculated value, once again, is below $-100 \mathrm{~dB}$. As the test point moves away from zenith, the $H$-plane cross-polarized component increases symmetrically and reaches its maximum at $\pm 90^{\circ}$.

Fig. 6(a) depicts a rectangular patch capacitively coupled to a coplanar transmission line [13]. $E$ - and $H$-plane radiation patterns are shown in Fig. 6(b). The substrate size is assumed to be three times the extent of the patch in both directions.

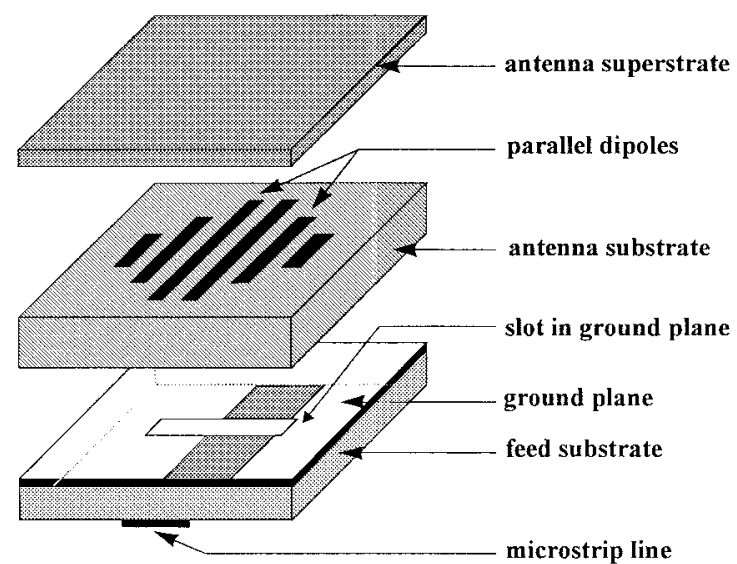

Fig. 7. Expanded view of multilayer microstrip antenna [1] comprised of parallel dipoles aperture coupled to a microstrip line.

In order to be able to model the fine features on the back side, nonequidistant segmentation was applied to the structure. Agreement between measurement [13] and computation is generally good. Notice the excellent agreement at $-180^{\circ}$, i.e., the direction facing the coplanar feed line.

A microstrip antenna composed of parallel dipole resonators of different lengths, aperture-coupled to a microstrip line (as suggested in [1]) is illustrated in Fig. 7. In order to be able to perform a meaningful calculation on available computers, we have modeled the radiating system as a threelayer assemblage only, assuming all metallic surfaces of zero thickness. Refraction occurring at the boundary of dielectrics was properly accounted for by means of Snell's Law. However, if the ray path (the electrical line of sight) for any two interacting currents was intercepting a metallic surface, mutual impedances for the two currents were set to zero; that is, we assumed that the metallic surfaces provide perfect shielding, i.e., have infinitely high conductivity. These two compromises were the most reasonable arrangement under the limitations of finite virtual memory and computer power. In addition, like in the structure of Fig. 6, a nonequidistant cellular structure was employed.

$E$ - and $H$-plane radiation patterns of the entire structure at $5.24 \mathrm{GHz}$ are presented in Fig. 8(a) and (b), respectively. The frequency corresponds to the resonance of the longest pair of dipoles (c.f., Fig. 7). Experimental data [1] are shown to be in good agreement with computed results, especially in the forward direction (angular variable in the range from $-90^{\circ}$ to $+90^{\circ}$ ) with respect to the radiators. We attribute the backwardradiation deviations to the aforementioned compromises that we had to make in the modeling process.

The computation time for this structure was $26 \mathrm{~h}$ on a SPARC station 20 with four $100 \mathrm{MHz}$ CPU's. All other calculations have been performed on an IBM RISC 6000/530 with CPU times ranging from 2 to $24 \mathrm{~h}$.

Note that all examples presented in this paper include the effect of the finite-size substrates and ground planes. A direct comparison between finite and infinite ground planes cannot be conducted with this method due to the limitations in computer resources. From a circuit (not pattern) point of view, 


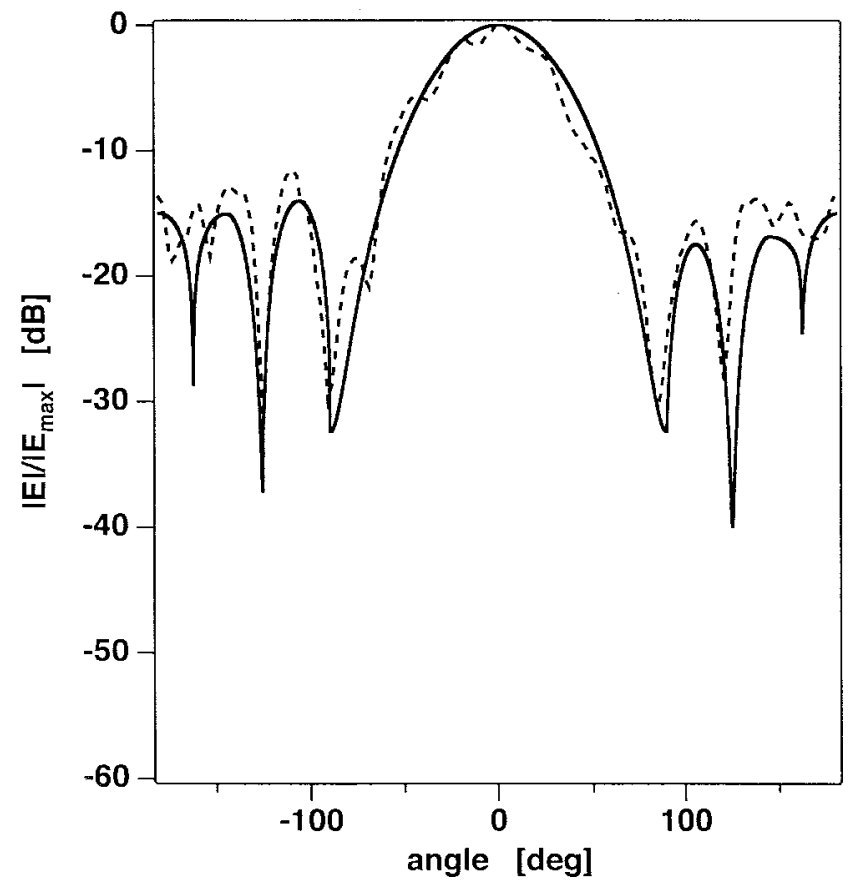

(a)

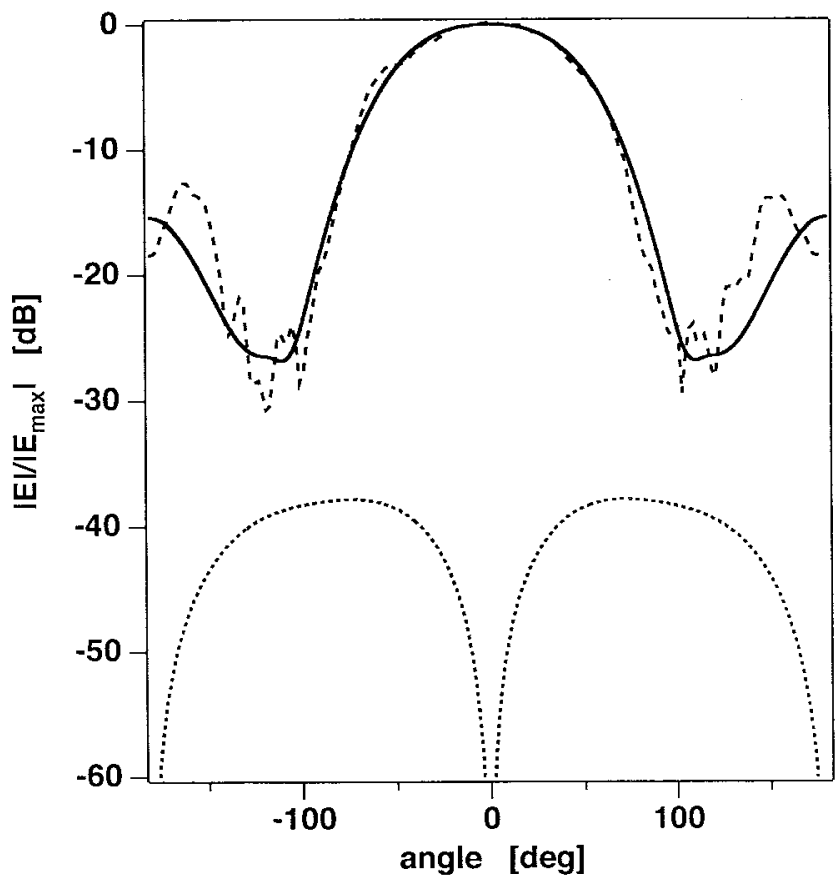

(b)

Fig. 8. Radiation patterns of the antenna of Fig. 7 at $5.24 \mathrm{GHz}$. Dimensions according to [1]. (a) $E$-plane-solid line: calculated copolarized; dashed line: measured copolarized [1]; calculated cross polarized below $-100 \mathrm{~dB}$. (b) $H$-plane-solid line: calculated copolarized; dashed line: measured copolarized [1]; dotted line: calculated cross polarized.

however, the influence of the finite-extent ground plane has been investigated in [8] and need not be repeated here. For a patch on GaAs substrate with two orthogonal feed lines and the ground-plane size only slightly larger than the radiating patch, the parameter most sensitive to a ground-plane size variation is the input reflection coefficient through the shift in resonance frequency. For further details, the reader is referred to [8].

\section{CONCLUSIONS}

A cellular-space-division-based MoM technique for the analysis of imperfect planar radiators and transmission lines on lossy substrates and finite-extent ground planes is presented. The surface impedances, which are associated with the equivalent surface currents defined on the thin-wall cells, represent their material properties. Dielectric and conductor losses are readily incorporated. Although effects due to radiation are fully included, the surrounding environment need not be modeled as material parameters are defined in relation to a homogeneous medium, e.g., free-space. Performance of the method in terms of radiation patterns of planar radiators is demonstrated at four examples with different feeding mechanisms. Good agreement with measured results is obtained. Aspects of built-in numerical integration techniques are discussed.

\section{ACKNOWLEDGMENT}

The authors would like to thank Prof. W. Menzel and his team at the Microwave Department, University of Ulm, Germany, for helpful suggestions and their support for the verification of experimental and theoretical results.

\section{REFERENCES}

[1] F. Croq and D.M. Pozar, "Multifrequency operation of microstrip antennas using aperture coupled parallel resonators," IEEE Trans. Antennas Propagat., vol. 40, pp. 1367-1374, Nov. 1992.

[2] Z. Cai and J. Bornemann, "Rigorous analysis of radiation properties of lossy patch resonators on complex anisotropic media and lossy ground metallization," IEEE Trans. Antennas Propagat., vol. 42, pp. 1443-1446, Oct. 1994.

[3] A. A. Kishk, M. R. Zunoubi, and D. Kajfez, "A numerical study of a dielectric disk antenna above grounded dielectric substrate," IEEE Trans. Antennas Propagat., vol. 41, pp. 813-821, June 1993.

[4] A. A. Kishk and L. Shafai, "The effect of various parameters of circular microstrip antennas on their radiation efficiency and the mode excitations," IEEE Trans. Antennas Propagat., vol. AP-34, pp. 969-976, Aug. 1986.

[5] B. J. Rubin and S. Daijavad, "Radiation and scattering from structures involving finite-size dielectric regions," IEEE Trans. Antennas Propagat., vol. 38, pp. 1863-1873, Nov. 1990

[6] M. Gimersky, S. Amari, and J. Bornemann, "Tuning-free analysis of planar radiators based on an exact numerical evaluation of the twodimensional generalized exponential integral," in IEEE AP-S Int. Symp. Dig., Newport Beach, CA, June 1995, pp. 1316-1319.

[7] _ "Numerical evaluation of the two-dimensional generalized exponential integral," IEEE Trans. Antennas Propagat., vol. 44, pp. $1422-1425$, Oct. 1996.

[8] M. Gimersky and J. Bornemann, "A modified method-of-moments technique for the full-wave analysis of imperfect conductors on lossy and finite-extent substrates," in IEEE MTT-S Int. Microwave Symp. Dig., San Francisco, CA, June 1996, pp. 715-718.

[9] C. A. Balanis, Advanced Engineering Electromagnetics. New York: Wiley, 1989, ch. 7

[10] C. A. McDonach, D. Nguyen, G. S. Gupta, and A. P. Luscombe, "Design of a dual polarized microstrip patch array for an advanced SAR antenna," in IGARSS Int. Geosci. Remote Sensing Symp. Dig., Vancouver, Canada, 1989, pp. 2275-2277.

[11] Private communication, Spar Aerospace Ltd., Ste. Anne-de-Bellevue, Quebec, Canada

[12] E. Chang, S. A. Long, and W. F. Richards, "An experimental investigation of electrically thick rectangular microstrip antennas," IEEE Trans. Antennas Propagat., vol. AP-34, pp. 767-772, June 1986.

[13] W. Menzel and W. Grabherr, "A microstrip patch antenna with coplanar feed line," IEEE Microwave Guided Wave Lett., vol. 1, pp. 340-342, Nov. 1991. 


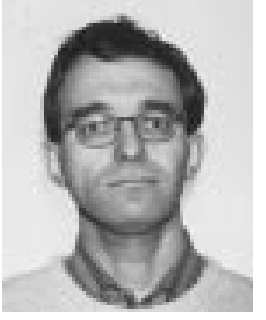

Martin Gimersky (S'91-M'97) was born in Presov, Czechoslovakia, on May 23, 1965. He received the Eng. degree from the Faculty of Electrical Engineering, Czech Technical University, Prague, Czechoslovakia, in 1988, and the Ph.D. degree in electrical engineering from the University of Victoria, Victoria, BC, Canada, in 1996.

From April to August 1996, he worked as a Postdoctoral Fellow at the University of Manitoba, Winnipeg, MB, Canada. Since September 1996, he has been on the technical staff of Spar Aerospace Limited, Ste. Anne-de-Bellevue, PQ, Canada. His main interests are in spaceborne antennas, particularly reflector antennas and their feeds, and numerical modeling in electromagnetics.

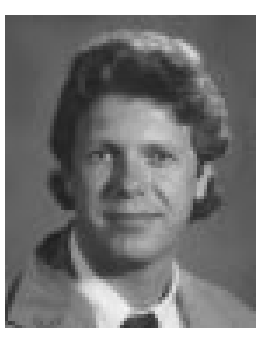

Jens Bornemann (M'87-SM'90) received the Dipl.-Ing. and the Dr.-Ing. degrees, both in electrical engineering, from the University of Bremen, Germany, in 1980 and 1984, respectively.

From 1984 to 1985 , he worked as a Consulting Engineer. In 1985 he joined the University of Bremen, Germany, as an Assistant Professor. Since April 1988 he has been with the Department of Electrical and Computer Engineering, University of Victoria, Victoria, B.C., Canada, where he became a Professor in 1992. In 1996, he was a Visiting Scientist at Spar Aerospace Limited, Ste. Anne-de-Bellevue, Québec, Canada, and a Visting Professor at the Microwave Department, University of Ulm, Germany. His research activities include microwave/millimeter-wave components and systems design and problems involving electromagnetic field theory in integrated circuits, waveguide feed networks, and radiating structures. He is a coauthor of Waveguide Components for Antenna Feed Systems-Theory and Design (Norwood, MA: Artech House, 1993) and has authored or coauthored more than 130 technical papers. He serves on the editorial board of the International Journal of Numerical Modeling.

Dr. Bornemann was one of the recipients of the A.F. Bulgin Premium of the Institution of Electronic and Radio Engineers in 1983. He is a Registered Professional Engineer in the Province of British Columbia, Canada, and has been a Fellow of the British Columbia Advanced Systems Institute (1992-1995). He is a Senior Member of the IEEE Microwave Theory and Techniques and Antennas and Propagation Societies and serves on the editorial boards of the IEEE Transactions ON MicrowaVe TheORY AND TeChNiques. 